Recepción: 18 / 12 / 2017

Aceptación: 26 / 02 / 2018

Publicación: 02 / 07 / 2018

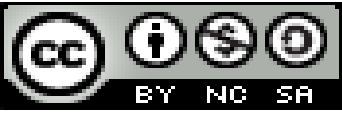

Ciencias de la salud

Artículo de Investigación

\title{
Anemia en el adulto mayor
}

\author{
Anemia in the elderly
}

\section{Anemia em idosos}

Jorge A. Cañarte-Alcivar ${ }^{\mathrm{I}}$ j.canarte.a@gmail.com

Elsa N. Lucas-Parrales II mnselsanoralma@hotmail.com

Mónica Y. Guerrero-Madroñero ${ }^{\mathrm{III}}$ monipucca22@hotmail.com

Ruth Y. Moreira-Vinces IV ruth.moreira@hotmail.com

\section{Correspondencia: j.canarte.a@gmail.com}

\footnotetext{
I Magister en Investigación Clínica y Epidemiológica, Especialista en Inmunología Clínica, Doctor en Medicina y Cirugía, Docente de la Universidad Técnica de Manabí, Portoviejo, Ecuador.

II Magister en Microbiología Mención Biomédica, Magister en Investigación Clínica y Epidemiológica, Diploma Superior en Microbiología, Licenciada en la Especialización de Laboratorio Clínico, Docente de la Universidad Estatal del Sur de Manabí, Jipijapa, Ecuador.

III Master en Docencia Universitaria, Médico Cirujano, Unidad Ambulatoria IESS Los Esteros, Manta, Ecuador.

IV Magister en Investigación Clínica y Epidemiológico, Licenciada en la Especialización de Laboratorio Clínico, Tecnólogo Médico Especialización de Laboratorio Clínico, Docente de la Universidad Laica Eloy Alfaro de Manabí, Manta, Ecuador.
} 


\title{
Resumen
}

La anemia es un problema frecuente en las personas mayores y está relacionada con un aumento de la morbilidad y mortalidad. Se realizó un estudio observacional, descriptivo y transversal, con la finalidad de caracterizar la anemia en los adultos mayores, según algunas variables de interés para la investigación. En la serie predominó el sexo masculino, de 60-69 años (65.5\%), la fatiga como síntoma principal (60.9\%), la anemia microcítica según el estudio de la lámina periférica, así como la anemia asociada a trastornos crónicos. A modo de conclusión, se debe incrementar el estudio del tipo de anemia en el adulto mayor para poder accionar ante el fenómeno del envejecimiento en América Latina y estudiar preventivamente a este grupo poblacional para diagnosticar afecciones crónicas e indicar tratamiento oportunamente.

Palabras clave: salud; anemia; envejecimiento; adulto mayor.

\begin{abstract}
Anemia is a common problem in older people and is related to an increase in morbidity and mortality. An observational, descriptive and transversal study was carried out in order to characterize the anemia in the elderly, according to some variables of interest for the investigation. In the series, the male sex predominated, 60-69 years (65.5\%), fatigue as the main symptom $(60.9 \%)$, microcytic anemia according to the study of the peripheral lamina, as well as anemia associated with chronic disorders. By way of conclusion, the study of the type of anemia in the elderly should be increased in order to be able to act on the aging phenomenon in Latin America and preventively study this population group to diagnose chronic conditions and indicate timely treatment.
\end{abstract}

Keywords: health; anemia; aging; elderly.

\section{Resumo}

A anemia é um problema comum em idosos e está relacionada ao aumento da morbidade e mortalidade. Foi realizado um estudo observacional, descritivo e transversal para caracterizar a anemia em idosos, segundo algumas variáveis de interesse para a investigação. Na série, predominou o sexo masculino, 60-69 anos (65.5\%), fadiga como principal sintoma (60.9\%), anemia microcítica de acordo com o estudo da lâmina periférica, além de anemia associada a distúrbios crônicos. A título de conclusão, o estudo do tipo de anemia em idosos deve ser 
aumentado para poder atuar sobre o fenômeno do envelhecimento na América Latina e estudar preventivamente esse grupo populacional para diagnosticar condições crônicas e indicar tratamento oportuno.

Palavras chave: saúde; anemia; envelhecimento idoso.

\section{Introducción}

La anemia es un problema de salud muy frecuente en los adultos mayores, se incrementa a partir de los 50 años. ${ }^{1}$ La prevalencia de anemia en los adultos mayores de 65 años asciende a $10 \%$ en las mujeres y $11 \%$ en los hombres, y aumenta $26.1 \%$ en hombres, y $20.1 \%$ en mujeres en los adultos mayores de 85 años. $^{2}$ Algunos estudios muestran que la prevalencia de anemia en los adultos mayores es variada y fluctúa entre 2.9 a $61 \%$ en los varones y 3.3 a $41.0 \%$ en las mujeres. ${ }^{3}$

La anemia se incrementa con la edad ${ }^{4-5}$ y está asociada con cambios en los estilos de vida, además de incrementar el riesgo por caídas, producir infecciones, disminuir el estado cognoscitivo y la capacidad funcional. Con frecuencia la anemia tiende a ser subdiagnósticada, debido a la coexistencia con alguna enfermedad ${ }^{9}$. Las causas de la anemia en los adultos mayores pueden ser clasificadas en tres categorías: deficiencia de la ingesta de hierro en la dieta, anemia asociada a enfermedades renales crónicas y la anemia no explicada. Esta última se atribuye a la disminución de los niveles de hemoglobina, posiblemente por la respuesta eritropoyética en la deficiencia de hierro; altos niveles de citoquinas proinflamatorias; la disminución de los niveles de andrógenos, y disminución proliferativa y regenerativa de las células de la médula ósea, mielodisplasia que está asociada con la disminución de la expectativa de vida. ${ }^{6}$

Se sabe que en los adultos mayores la anemia compromete las aptitudes psicofísicas y aumenta la morbimortalidad. En estas personas la presencia de anemia se relaciona con disminución de las funciones motrices y sensoriales, caídas, fragilidad, demencia, hospitalización y mortalidad. ${ }^{7}$

Teniendo en cuenta el criterio de la Organización Mundial de la Salud (OMS) para el diagnóstico de la anemia, se considera que en este grupo poblacional existe anemia cuando la cifra de hemoglobina es de $11 \mathrm{~g} / \mathrm{L}$ o inferior a esta, tanto en uno u otro sexo. En algunos de estos pacientes, cuando no existe una explicación para este trastorno, entonces se le denomina con el simple término de anemia senil, que debe considerarse inadecuado. La anemia que aparece en el

\section{4}


adulto mayor no obedece al envejecimiento, sino que es consecuencia de un número elevado de enfermedades anemizantes. ${ }^{8}$

Las causas de la anemia en los adultos mayores pueden ser clasificadas en 3 categorías, a saber: deficiencia de la ingesta de hierro en la dieta, asociada a enfermedades renales crónicas y la anemia no explicada. ${ }^{6}$

La prevalencia de anemia en el anciano varía. Según los diferentes estudios oscila entre 9-11\% para la población de 65 años o más; se duplica en los mayores de 85, principalmente en el sexo masculino y se cuadriplica en los ancianos institucionalizados. ${ }^{9}$

A pesar de ser la anemia una afección que puede aparecer en cualquier grupo poblacional, es importante el estudio de la misma en este grupo etéreo, por lo que se considera oportuno realizar esta investigación con vistas a caracterizar a los adultos mayores con este trastorno.

\section{Metodología}

Se realizó un estudio observacional, descriptivo y transversal, con la finalidad de caracterizar la anemia en los adultos mayores en el periodo de febrero del 2016 a febrero 2017.

La población que se estudió, estuvo conformada por 87 ancianos, los cuales acudieron a consulta, para caracterizarles según algunas variables de interés para la investigación. Los gerontes presentaron niveles de hemoglobina de $11 \mathrm{~g} / \mathrm{L}$ o inferior a esta cifra, a los cuales se les realizaron, previo consentimiento informado, exámenes complementarios (hemoglobina y lámina periférica). Se tuvieron en cuenta determinados criterios de inclusión y de exclusión.

Tanto a los pacientes como a los familiares se les informó sobre los detalles del estudio y se garantizó autonomía, justicia, beneficencia y no maleficencia. Los datos fueron utilizados con fines científicos.

Como instrumento, se aplicó encuesta confeccionada al efecto en la consulta. También se revisaron las historias clínicas por jefe de la investigación, para completar la información que no pudo ser obtenida a través de la aplicación de la encuesta.

Se utilizó el porcentaje como medida de resumen. 


\section{Resultados}

En la serie estudiada (tabla 1), predominaron el sexo masculino (65.5\%) y el grupo etario de 6069 años $(60.9 \%)$.

Tabla 1. Gerontes con anemia según edad y sexo.

\begin{tabular}{|c|c|c|c|c|c|c|}
\hline \multirow{2}{*}{$\begin{array}{c}\text { Grupo de } \\
\text { edades }\end{array}$} & \multicolumn{2}{|c|}{ Masculino } & \multicolumn{2}{|c|}{ Femenino } & \multicolumn{2}{|c|}{ Total } \\
\hline & No & $\%$ & No & $\%$ & No & $\%$ \\
\hline $60-69$ & 32 & 56.1 & 21 & 70.0 & 53 & 60.9 \\
\hline $70-79$ & 16 & 28.1 & 4 & 13.3 & 20 & 22.9 \\
\hline $80-89$ & 8 & 14.0 & 2 & 6.6 & 10 & 11.4 \\
\hline+90 & 1 & 1.7 & 3 & 10 & 4 & 4.5 \\
\hline Total & 57 & 65.5 & 30 & 34.4 & 87 & 100 \\
\hline
\end{tabular}

Como se muestra en la tabla 2, la mayoría de los afectados (71.2\%) manifestaron que no satisfacían sus necesidades básicas.

Tabla 2. Gerontes según satisfacción de las necesidades básicas.

\begin{tabular}{|c|c|c|c|c|c|c|}
\hline \multirow{2}{*}{$\begin{array}{l}\text { Satisfacción de las } \\
\text { necesidades básicas }\end{array}$} & \multicolumn{2}{|c|}{ Masculino } & \multicolumn{2}{|c|}{ Femenino } & \multicolumn{2}{|c|}{ Total } \\
\hline & No & $\%$ & No & $\%$ & No & $\%$ \\
\hline Buena & 18 & 20.6 & 7 & 38.8 & 25 & 28.7 \\
\hline Mala & 51 & 73.9 & 11 & 61.1 & 62 & 71.2 \\
\hline Total & 69 & 79.3 & 18 & 20.6 & 87 & 100 \\
\hline
\end{tabular}


Entre las manifestaciones clínicas preponderantes, figuraron la fatiga - debilidad en los grupos de 80-89 años y de 90 y más, respectivamente, seguidas de la palidez y vértigos (tabla 3).

Tabla 3. Manifestaciones clínicas más frecuentes.

\begin{tabular}{|l|c|c|}
\hline Manifestaciones clínicas & No & \% \\
\hline Vértigo & 20 & 22.9 \\
\hline Palidez cutánea - mucosa & 40 & 45.9 \\
\hline Fragilidad mental - emocional & 11 & 12.6 \\
\hline Fatiga - debilidad & 53 & 60.9 \\
\hline Dolores óseos & 8 & 9.1 \\
\hline
\end{tabular}

Al estudiar las principales alteraciones, según el resultado de la lámina periférica (tabla 4), se observó el predominio de la anemia microcítica en las féminas (57.4\%), seguida en orden de frecuencia por la macrocítica $(22.9 \%)$.

Tabla 4. Principales alteraciones según resultado de lámina periférica

\begin{tabular}{|l|c|c|}
\hline $\begin{array}{c}\text { Alteraciones según resultado de } \\
\text { lámina periférica }\end{array}$ & No & \% \\
\hline Macrositicas & 20 & 22.9 \\
\hline Normociticas & 15 & 17.2 \\
\hline Microciticas & 50 & 57.4 \\
\hline
\end{tabular}

Según las causas de la anemia, sobresalieron las asociadas con trastornos crónicos (57.7\%), con predominio no significativo del sexo masculino.

Al analizar las condiciones de vida de los gerontes, más del 50.0\% vivían en zona urbana, el 22.0\% eran analfabetos, y el nivel de pobreza poco extremo. Con respecto a la seguridad social, el per cápita es inferior para cubrir sus necesidades básicas.

\section{Discusión}


El envejecimiento de la población a nivel mundial avanza progresivamente ${ }^{10}$; tanto en los países desarrollados como en vías de desarrollo, sin embargo, la preparación o forma como se afronta este periodo, difiere entre los adultos mayores si residen en uno u otro lugar. La mayoría de estudios publicados en adultos mayores fueron realizados en los ambientes hospitalarios. Son muy escasos los estudios de anemia en adultos mayores con base poblacional.

El incremento de la esperanza de vida de la población y la culminación de la transición epidemiológica ha traído consigo un aumento progresivo de los pacientes de edad avanzada, tanto en términos absolutos como relativos. La característica comorbilidad de los pacientes ancianos ha conducido a una aproximación multidisciplinar que implica mayor interacción de la geriatría con otras especialidades, como la hematología.

Así mismo, investigación realizada por Tarqui Mamani et al. ${ }^{6}$ demostró asociación entre el sexo masculino y la anemia, aunque el análisis multivariado no mostró asociación significativa, estos resultados difieren de lo reportado por Bang et al., ${ }^{12}$ que refieren que las mujeres presentan mayor prevalencia de anemia que los varones.

Algunos autores ${ }^{6}$ encontraron en su estudio que, en los adultos con más de 65 años la anemia asciende a $10 \%$ en las mujeres y a $11 \%$ en los hombres; mientras que en los mayores de 85 aumenta 26.1 y $20.1 \%$ en hombres y mujeres, respectivamente.

Los adultos mayores con anemia tienen más fragilidad tanto física como mental y emocionalmente. Las consecuencias podrían variar según la gravedad de la anemia. En nuestro estudio más el 12.6\% de los gerontes manifestaron fragilidad tanto física como mental. ${ }^{13}$

En los últimos años, grandes estudios epidemiológicos se han documentado que la existencia de anemia en el anciano, aunque no sea grave, es un factor predictivo de mal pronóstico funcional y vital a medio-largo plazo. Esta afirmación es válida tanto para ancianos aparentemente sanos, como para aquellos que cumplen criterios de fragilidad. De hecho, se ha demostrado una fuerte asociación entre anemia y las características fenotípicas del síndrome de fragilidad como sarcopenia, reducción de la fuerza muscular y problemas de movilidad. Asimismo, estudios epidemiológicos han documentado que la anemia leve (criterios OMS) es factor predictivo de fragilidad. ${ }^{14}$ 
En un estudio realizado en Lima, Perú, ${ }^{6}$ se obtuvo que la satisfacción de necesidades básicas fue de forma regular o mala, lo cual coincide con lo descrito en esta serie. Es importante señalar que en esta etapa de la vida el adulto mayor se jubila, en esta etapa de la vida se incrementan las enfermedades crónicas y con esto la necesidad de comprar medicamentos, sin dejar de mencionar la polifarmacia que es frecuente en este grupo etario, de modo que priorizan estas situaciones y desplazan la calidad de la alimentación a otro nivel prioritario. En el resto de la bibliografía médica revisada no se encontraron estudios que compararan esta variable, por lo cual estos resultados incitan a estudiarla con más sistematicidad.

Más de 1000 millones de personas, poseen alguna forma de déficit de hierro y unos 500 millones tienen anemia ferropenia; sin embargo, existen grandes diferencias entre las regiones pobres y ricas del mundo. Así, en los países en vías de desarrollo oscila entre 2-28\%. En España, la prevalencia en lactantes y preescolares es de 5.7\%; en varones adultos y en mujeres que no menstrúan, de 1.6 $\%$, respectivamente. ${ }^{11}$

La anemia no explicada se atribuye a la disminución de los niveles de hemoglobina, posiblemente por la respuesta eritropoyética en la deficiencia de hierro, a los altos niveles de citoquinas proinflamatorias, a la disminución de los niveles de andrógenos, así como a la disminución proliferativa y regenerativa de las células de la médula ósea; mielodisplasia que está asociada con la disminución de la expectativa de vida. ${ }^{11}$

Comúnmente, las anemias normocíticas en los ancianos obedecen a una causa inflamatoria o asociada a insuficiencia renal; asimismo, la macrocitosis acompaña a la anemia por déficit de folatos y cobalamina, incluso, las precede semanas o meses. Si en estas circunstancias no hay macrocitosis, debe sospecharse la coexistencia de un trastorno microcítico, como ferropenia o rasgo talasémico. En nuestro estudio prevaleció la micrositicas, coincidiendo con los resultados de González KÁ et al. ${ }^{11}$

Resulta de particular importancia identificar y tratar a los pacientes con anemia y no considerar esta enfermedad como una consecuencia inevitable de la vejez. Por este motivo, los ancianos están expuestos frecuentemente a tratamientos.

Se concluye, que la jubilación muchas veces ocasiona insatisfacción de las necesidades básicas y envejecimiento individual, dado el incremento de afecciones que demandan mayor sustento 
económico; asimismo, los síntomas más frecuentes encontrados son inespecíficos de la anemia, pues pueden existir enfermedades crónicas asociadas que enmascaran esta enfermedad.

\section{Referencias Bibliográficas}

1. Ania BJ, Suman VJ, Fairbanks VF, Rademacher DM, Melton LJ 3rd. Incidence of anemia in older people: an epidemiologic study in a well defined population. J Am Geriatr Soc. $1997 ; 45(7): 825-831$

2. Guralnik JM, Eisenstaedt RS, Ferrucci L, Klein HG, Woodman RC. Prevalence of anemia in persons 65 years and older in the United States: evidence for a high rate of unexplained anemia. Blood. 2004;104(8):2263-8.

3. Beghe C, Wilson A, Ershler W. Prevalence and outcomes of anemia in geriatrics a systematic review of the literature. Am J Med. 2004;116 Suppl 7A:3S-10S

4. Gabrilove J. Anemia and the elderly: clinical considerations. Best Pract Res Clin Haematol. 2005;18(3):417-22.

5. Andres E, Federici L, Serraj K, Kaltenbach G. Update of nutrient-deficiency anemia in elderly patients. Eur J Intern Med. 2008;19(7):488-93. doi: 10.1016/j.ejim.2008.01.016

6. Tarqui Mamani C, Sanchez Abanto J, Álvarez Dongo D, Espinoza Oriundo P, Jordan Lechuga T. Prevalencia de anemia y factores asociados en adultos mayores peruanos. Rev Peruana Med Exp Salud Pública. 2015 [citado 13 mayo 2017]; 32(4). Disponible en: http://www.scielo.org.pe/scielo.php?pid=S1726$46342015000400009 \&$ script=sci_arttext\&tlng=pt

7. Cappellini MD, Motta I. Anemia in clinical practice-denition and classication: Does Hemoglobin change with aging? Semin Hematol 2015; 52: 261-9.

8. Osorio G, Barrientos G. Anemia en el adulto mayor. En: Osorio Barrientos G. Ciencia y medicina [citado 13 mayo 2017]. Disponible en: http://www.medicinayhumanidades.cl/ ediciones/n12009/05_Ciencia\%20y\%20Medicina.pdf

9. Vidales Hernández M de, Mao Martín L, García Panadés R, Díaz Sanchez J. Anemia en paciente anciano ¿podemos estar tranquilos? Revista SEMERGE. Medicina de familia. 2011 
[citado 13 mayo 2017]; 37(7). Disponible en: http://www.elsevier.es/es-revista-semergen medicina-familia-40-articulo-anemia-paciente-anciano-podemos-estar-S1138359311000682

10. Organización de las Naciones Unidas. Segunda Asamblea Mundial Sobre el Envejecimiento. Madrid: ONU; 2002.

11. González KÁ, Madera Aguia Y, Díaz Calzada M, Naranjo Ferregut JA. Caracterización de adultos mayores con anemia. MEDISAN. 2017 [citado 2017 Dic 16]; 21(11): [aprox. 0 p.]. Disponible en: http://www.medisan.sld.cu/index.php/san/article/view/1562

12. Bang SM, Lee JO, Kim YJ, Lee KW, Lim S, Kim JH, et al. Anemia and activities of daily living in the Korean urban elderly population: Results from the Korean Longitudinal Study on Health and Aging (KLoSHA). Ann Hematol. 2013;92(1):59-65. doi: 10.1007/s00277-012- 1563-6

13. Adultos mayores son la población con más anemia en Costa Rica. 2012. [citado 2017 May 16]. Disponible en: https://www.nacion.com/ciencia/salud/adultos-mayores-son-la-poblacion- conmas-anemia-en-costa-rica/TOBRZZ2NH5HVVK3FPYWDWMVKOQ/story/

14. Urrutia A, Sacanella E, Mascarod J, Formiga F. Anemia en el anciano. Rev Esp Geriatr Gerontol 2010; 45:291-7 - DOI: 10.1, 6\&lng=es 